UCRL-JC-121454

PREPRINT

\title{
IRON FREE PERMANENT MAGNET SYSTEMS FOR CHARGED PARTICLE BEAM OPTICS
}

\author{
S.M. Lund and K. Halbach ${ }^{+}$ \\ Lawrence Livermore National Laboratory, University of California \\ P.O. Box 5508, L-440, Livermore California 94550, USA \\ ${ }^{+}$Lawrence Berkeley Laboratory, University of California \\ 1 Cyclotron Road, Berkeley California 94720, USA
}

This paper was prepared for submittal to the

Proceedings of the International Symposium On Heavy Ion Fusion

Princeton, New Jersey

September 6-9, 1995

September 3, 1995

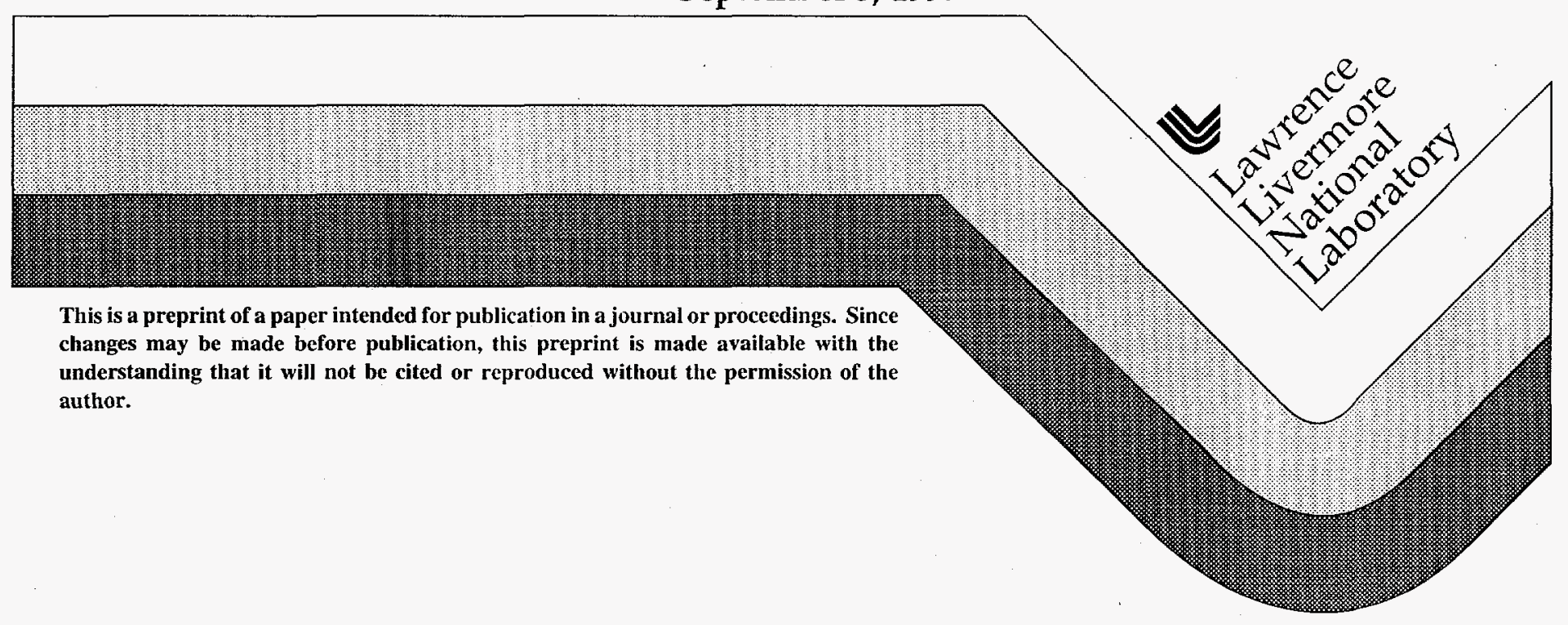




\section{DISCLAIMER}

This document was prepared as an account of worksponsored by an agency of the United States Government. Neither the United States Government nor the University of California nor any of their employees, makes any warranty, express or implied, or assumes any legal liability of responsibility for the accuracy, completeness, or usefulness of any information, apparatus, product, or process disclosed, or represents that its use would not infringe privately owned rights. Reference herein to any specific commercial products, process, or service by trade name, trademark, manufacturer, or otherwise, does not necessarily constitute or imply its endorsement, recommendation, or favoring by the United States Government or the University of California. The views and opinions of authors expressed herein do not necessarily state or reflect those of the United States Government or the University of California, and shall not be used for advertising or product endorsement purposes. 


\section{DISCLAMMER}

Portions of this document may be illegible in electronic image products. Images are produced from the best available original document. 


\title{
Iron free permanent magnet systems for charged particle beam optics
}

\author{
Steven M. Lund \\ University of California, Lawrence Livermore National Laboratory, Livermore, California 94550 , \\ $U S A$ \\ Klaus Halbach \\ University of California, Lawrence Berkeley National Laboratory, Berkeley, California 94720 , \\ $U S A$
}

\begin{abstract}
The strength and astounding simplicity of certain permanent magnet materials allow a wide variety of simple, compact configurations of high field strength and quality multipole magnets. Here we analyze the important class of iron-free permanent magnet systems for charged particle beam optics. The theory of conventional segmented multipole magnets formed from uniformly magnetized block magnets placed in regular arrays about a circular magnet aperture is reviewed. Practical multipole configurations resulting are presented that are capable of high and intermediate aperture field strengths. A new class of elliptical aperture magnets is presented within a model with continuously varying magnetization angle. Segmented versions of these magnets promise practical high field dipole and quadrupole magnets with an increased range of applicability.
\end{abstract}




\section{INTRODUCTION}

Permanent magnet (PM) lenses for charged particle optics are now recognized in many applications as being practical, cost-effective alternatives to conventional electromagnet and superconducting magnet lenses [1]. High-quality and high-field PM dipole, quadrupole, and sextupole multipole magnets are now in wide use [1]. A fundamental advantage of PM systems is that the aperture field strength remains invariant as the linear dimensions of the system are scaled [2]. On the other hand, for fixed field strength in conventional magnets, the current density in the coils must scale inversely proportional to the linear magnet dimensions. Thus, compact, high-gradient PM lenses are attainable in parameter regimes where cooling problems can preclude the use of conventional electromagnets. Furthermore, PM systems eliminate the additional complexity of external power supplies, control systems, plumbing for cooling, etc., needed in conventional magnet systems. The increasingly important subclass of iron-free PM systems are characterized by simple, compact, high field strength and quality structures with remarkably simple fringe field properties $[2,4]$. The applicability of linear superposition in such systems renders analytical design tractable, thereby enabling scaling laws, design innovations, and physical insights to be readily obtained. Moreover, linear superposition allows, if necessary, the insertion of these magnets into conventional current dominated magnets and other iron-free PM systems to provide some degree of field variability.

As a practical matter, iron-free PM multipole lenses can provide an attractive alternative to conventional magnetic lenses in situations where adjustability is not strictly necessary, clear beam apertures are moderate (circular aperture radii on the order of 5 centimeters or less), and maximum aperture field strengths are well within materials limits. For the important cases of dipole and quadrupole lenses, these aperture field limits for currently available designs and PM materials are of the order of 1 Tesla for dipoles and 2 Tesla for quadrupoles [2]. Generally speaking, smaller aperture fields and smaller apertures are

more economical, provided the aperture is not so small that materials tolerances become 
problematic.

\section{MATERIAL PROPERTIES AND THEORETICAL MODEL}

In this section we summarize needed theoretical results and practical material limits that are presented in detail in the references [2-4]. The reader is encouraged to refer to those references for further information.

We consider classes of PM materials such as rare-earth compounds of samarium-cobalt $(\mathrm{SmCo})$ and compounds of neodymium-iron-boron $(\mathrm{NdFeB})$. For such materials, the threedimensional (3D) field components of the magnetic induction $\mathbf{B}$ and the magnetic field $\mathbf{H}$ are related within the medium as $B_{\|}=B_{r}+\mu_{0} \mu_{\|} H_{\|}$and $\mathbf{B}_{\perp}=\mu_{0} \mu_{\perp} \mathbf{H}_{\perp}$ in directions parallel $(\|)$ and perpendicular $(\perp)$ to the crystalline axis (the so-called easy-axis) of the PM material. Both here and henceforth MKS units are employed, $\mu_{0}=4 \pi \times 10^{-7} \mathrm{Vs} / \mathrm{Am}$ is the permeability of free-space, $B_{r}$ is the so-called remanent field or intrinsic magnetization of the medium, and $\mu_{\|}$and $\mu_{\perp}$ are differential permeabilities parallel and perpendicular to the easy-axis. Both $\mu_{\|}$and $\mu_{\perp}$ are approximately unity over a wide range of field values $\left(\mu_{\|}-1 \sim \mu_{\perp}-1 \sim 0.02\right.$ to 0.05 typical) and the material characteristics can be approximated by the simple vector relation [2]

$$
\mathbf{B}=\mathbf{B}_{r}+\mu_{0} \mathbf{H}
$$

where $\mathbf{B}_{r}$ is a vector of magnitude $B_{r}$ parallel to the easy-axis in the medium and zero outside. Consequently, the fields produced by different pieces of PM material superimpose linearly. This property, together with the strong material strength reflected by remanent fields $B_{r}$ that can be as large as 1.35 Tesla and 1.0 Tesla for available NdFeB and SmCo materials, respectively [5], allows the construction of simple, compact, high-field multipole magnets.

As a practical matter, the ideal material behavior described above can be violated if $\mu_{0} H_{\|}$is driven significantly antiparallel to $B_{r}[2]$. This can result in nonlinear demagnetizing 
effects that damage the magnet. Such conditions must be avoided in both the operation and assembly of a practical design. Also, manufacturing techniques constrain the properties of available PM material. Currently, PM material is available in blocks of specific and special geometric shapes, but the smallest linear dimension of a block cannot be larger than a few centimeters. Further, blocks are uniformly magnetized (fixed easy-axis orientation and remanent field strength $B_{r}$ ). Large magnet blocks can be assembled from smaller blocks, but high PM materials cost and labor associated with making and handling a large number of individual components can lead to expensive magnet systems. Cost effective PM devices need a minimal number of distinct magnet blocks and have small magnet volume due to high material cost. Outside of high radiation environments, properly aged PM material exhibits good stability in time. The magnet strength is observed to undergo a slow logarithmic decay in time that is insignificant for many applications [6]. PM material is also temperature sensitive. The maximum temperature $T_{\max }$ of the medium should be kept a bit below the Curie temperature $T_{c}$ to avoid magnet damage $\left(T_{\max } \sim 100^{\circ} \mathrm{C}\right.$ and $T_{c} \sim 300^{\circ} \mathrm{C}$ for $\mathrm{NdFeB}$, and $T_{\max } \sim 250^{\circ} \mathrm{C}$ and $T_{c} \sim 700^{\circ} \mathrm{C}$ for $\mathrm{SmCo}$ ), precluding all but moderate vacuum baking. Aiso significant temperature coefficients $\left(\sim-0.1 \% /{ }^{\circ} \mathrm{C}\right.$ and $\sim-0.04 \% /{ }^{\circ} \mathrm{C}$ variation in $B_{r}$ for $\mathrm{NdFeB}$ and $\mathrm{SmCo}$, respectively) may necessitate operation in a stable temperature environment, and the local temperature of a magnet should not be varied rapidly in space or time. Finally, oriented ferrites have significantly lower cost and qualitatively similar material properties to those outlined above. However, a weaker maximum remanent field strength of $B_{r} \sim 0.42$ Tesla limits these materials to low-field applications in iron-free designs. Moreover, higher differential permeabilities associated with oriented ferrites $\left(\mu_{\|}-1 \sim \mu_{\perp}-1 \sim 0.1\right.$ typical) render simple analytic design of iron-free magnets more approximate, but does not complicate the design of iron-dominated magnets. Also, higher temperature coefficients $\left(\sim-0.3 \% /{ }^{\circ} \mathrm{C}\right.$ variation in $B_{r}$ typical) may necessitate greater care in the control of operating temperature.

The Maxwell equations $\nabla \times \mathbf{H}=0$ and $\nabla \cdot \mathbf{B}=0$, together with the materials equation (1) describe the 3D fields of the PM system. The curl equation and Eq. (1) can be combined 
as $\nabla \times \mathbf{B}=\nabla \times \mathbf{B}_{r}$, where $\mu_{0} \mathbf{j}=\nabla \times \mathbf{B}_{r}$ can be interpreted as an effective "current density" that characterizes the PM material. Thus, in the absence of iron, the fields produced by PM material can be thought of simply as resulting from an imprinted current density $\mu_{0} \mathbf{j}=\nabla \times \mathbf{B}_{r}$ in vacuum [2]. (Similar arguments can be used to show that $\mathbf{H}$ can be regarded as being due to an imprinted "charge density" $-\nabla \cdot \mathbf{B}_{r}$.) Furthermore, it is clear from the field equations that if all coordinates are scaled by $\alpha$, then $\mathbf{B}$ remains unchanged. This invariance proves the important property that the field strength of a PM system is independent of the actual physical size of the system, thereby allowing the possibility of very high gradient structures.

An iron-free PM multipole magnet can typically be regarded as an axial extrusion of a transverse magnetic structure over an axial length $\ell$. The applicability of linear superposition allows one to derive a rather remarkable property of the fringe fields of such a system [2]. We proceed as follows: Let $z$ and $\mathbf{x}_{\perp}$ be axial and transverse coordinates, and let $G$ be any 3D field component of the system. Make an infinite axial extrusion of the magnetic structure and denote $G^{2 d}$ as the field component $G$ in this two-dimensional (2D) limit. Referring to Fig. 1(a), we divide the magnet at $z=0$ into two semi-infinite sections. By symmetry, the field components $G^{+}\left(\mathrm{x}_{\perp}, z\right)$ and $G^{-}\left(\mathrm{x}_{\perp}, z\right)$ due to the sections with positive and negative axial coordinates are related as $G^{+}\left(\mathrm{x}_{\perp}, z\right)=G^{-}\left(\mathrm{x}_{\perp},-z\right)$, and their sum must equal the field component in the 2D limit, i.e., $G^{+}\left(\mathrm{x}_{\perp}, z\right)+G^{-}\left(\mathrm{x}_{\perp}, z\right)=G^{2 d}\left(\mathrm{x}_{\perp}\right)$. The fields deep within the semi-infinite magnets approach the limiting $2 \mathrm{D}$ values for $|z|>\ell_{0}$, with $\ell_{0}$. chosen sufficiently large. Then it follows immediately that $\int_{-\ell_{0}}^{\ell_{0}} d z G^{ \pm}\left(\mathrm{x}_{\perp}, z\right)=\ell_{0} G^{2 d}\left(\mathrm{x}_{\perp}\right)$. In addition, the value of $G^{ \pm}$at the entry planes of the semi-infinite magnets are exactly half the $2 \mathrm{D}$ limit value, i.e., $G^{ \pm}\left(\mathrm{x}_{\perp}, z=0\right)=G^{2 d}\left(\mathrm{x}_{\perp}\right) / 2$. Regard the physical magnet of length $\ell$ as a linear superposition of two semi-infinite magnets, magnet $\mathrm{A}$ and magnet $\mathrm{B}$, as sketched in Fig. 1(b). Magnet $A$ is an axial extrusion of the transverse magnet structure of the physical magnet, while magnet $\mathrm{B}$ is a translated axial extrusion of that structure with all remanent fields reversed. The remarkable result 


$$
\int_{-\infty}^{\infty} d z G\left(\mathbf{x}_{\perp}, z\right)=\ell G^{2 d}\left(\mathbf{x}_{\perp}\right)
$$

then follows immediately from linear superposition of the field integrals of the semi-infinite magnets obtained from the $\ell_{0} \rightarrow \infty$ limit of the previous integrals.

For any axially extruded iron-free PM magnet system, Eq. (2) immediately shows that the effective length of any field component defined in terms of the normalized axial integral $(1 / \ell) \int_{-\infty}^{\infty} d z \cdots$ of the field component is equal to the physical axial length of the magnet, and also that the axial integral of any field quantity vanishes if that field quantity is zero in the 2D limit. Therefore, the design of iron-free PM magnet systems can be effectively carried out within a transverse $2 \mathrm{D}$ model since results obtained are concretely related to the physical 3D systems of interest. Furthermore, within the 2D model, powerful analytic tools ease the development of physical insight. In the authors opinions, the utility of $2 \mathrm{D}$ modeling should not be underestimated. Henceforth, all analysis will refer to axially extruded ironfree PM systems. Finally, Eq. (2) and the consequences immediately following are not generally true for magnet systems with iron (PM or otherwise) or for conventional current magnets formed from macroscopic current loops. This follows because the simple linear superposition arguments leading to Eq. (2) are not valid in the presence of iron due to changing boundary conditions or in the presence of macroscopic current loops since such loops cannot be regarded as axially extruded structures due to the ends. However, Eq. (2) also remains valid if both the $2 \mathrm{D}$ and $3 \mathrm{D}$ fields are calculated in the presence of a sufficiently long axial iron shell of arbitrary transverse cross-section that surrounds the PM material.

For a PM system that is sufficiently long in the axial $z$-direction $(\ell \gg$ transverse magnet extent), the fields near the axial ( $z=0)$ midplane are approximately transverse $2 \mathrm{D}$ fields. Alternatively, it follows from Eq. (2) that the normalized axial integrals $(1 / \ell) \int_{-\infty}^{\infty} d z \cdots$ of the $3 \mathrm{D}$ field components are identically the $2 \mathrm{D}$ limit field components. We now present theoretical formulae for the calculation of $2 \mathrm{D}$ fields produced by PM material without notationally distinguishing between these cases. Results may be interpreted as preferred.

In vacuum regions, the $2 \mathrm{D}$ field components $B_{x}$ and $B_{y}$ along the Cartesian $x$ - and $y$ - 
coordinate axes can be derived from a magnetic scalar potential $\phi$ or the axial component of the vector potential $A$ as

$$
\begin{aligned}
& B_{x}=-\mu_{0} \frac{\partial \phi}{\partial x}=\frac{\partial A}{\partial y} \\
& B_{y}=-\mu_{0} \frac{\partial \phi}{\partial y}=-\frac{\partial A}{\partial x} .
\end{aligned}
$$

These can be considered as the Cauchy-Riemann conditions for the complex potential $\underline{F} \equiv$ $A+i \mu_{0} \phi$ of an analytical function of the complex variable $\underline{z}=x+i y$. It follows immediately that $\underline{B}^{*}=B_{x}-i B_{y}=i d \underline{F} / d \underline{z}$ is also an analytical function of $\underline{z}$ in vacuum regions and the power of the theory of an analytical function of a complex variable can be applied [2]. Here and henceforth, underlines denote complex variables, e.g., $\underline{B}=B_{x}+i B_{y}$, etc., $i=\sqrt{-1}$, and an asterisk (*) denotes complex conjugation. The complex coordinate $\underline{z}=x+i y$ should not be confused with the axial coordinate $z$, and it is often convenient to employ the polar form $\underline{z}=r e^{i \theta}$, where $r$ is the radius and $\theta$ is the azimuthal angle.

The $2 \mathrm{D}$ field of a current filament at location $\underline{z}^{\prime}=x^{\prime}+i y^{\prime}$ is given by $\underline{B}^{*}=\left(\mu_{0} / 2 \pi i\right) I /(\underline{z}-$ $\left.\underline{z}^{\prime}\right)$. Since the fields produced by PM material can be thought of as arising from an effective current density $\mu_{0} \mathbf{j}=\nabla \times \mathbf{B}_{r}$ in vacuum, we may superimpose the fields due to the $z$ component effective current filaments $\mu_{0} j_{z}=\partial B_{r y} / \partial x-\partial B_{r x} / \partial y$ to express the 2D field exterior to the medium as [2]

$$
\underline{B}^{*}[\underline{z}]=B_{x}-i B_{y}=\frac{1}{2 \pi i} \int d x^{\prime} d y^{\prime} \frac{\partial B_{r y} / \partial x-\partial B_{r x} / \partial y}{\underline{z}-\underline{z}^{\prime}} .
$$

Without restriction on the local remanent field $\underline{B}_{r}=B_{r x}+i B_{r y}$ of the magnet(s), this formula can be expressed as [2]

$$
\underline{B}^{*}[\underline{z}]=B_{x}-i B_{y}=\frac{1}{2 \pi} \int d x^{\prime} d y^{\prime} \frac{\underline{B}_{r}\left[\underline{z}^{\prime}\right]}{\left(\underline{\underline{z}}-\underline{z}^{\prime}\right)^{2}} .
$$

Equation (4) is used extensively in the analysis of 2D fields from iron-free PM systems. The important easy axis rotation theorem is an immediate consequence of Eq. (4). This theorem can be stated as follows [2]. If in an iron-free. 2D PM system all easy-axes are rotated by an angle $+\gamma$, then the local magnetic field outside the PM media rotates by an angle $-\gamma$, without change in amplitude. 
* If all PM material is contained within an annular region of inner radius $r_{i}$ and outer radius $s r_{i}$, with $s>1$, then Eq. (4) can be expanded within the interior $\left(|\underline{z}|<r_{i}\right)$ and exterior $\left(|\underline{z}|>s r_{i}\right)$ regions to obtain [2]

$$
\underline{B}^{*}[\underline{z}]= \begin{cases}\sum_{n=1}^{\infty} \underline{b}_{n} \underline{z}^{n-1}, & |\underline{z}|<r_{i} \\ \sum_{n=-1}^{-\infty} \underline{b}_{n} \underline{z}^{n-1}, & |\underline{z}|>s r_{i}\end{cases}
$$

where

$$
\underline{b}_{n}=\frac{n \operatorname{sgn}(n)}{2 \pi} \int d x d y \frac{\underline{B}_{r}[\underline{z}]}{\underline{z}^{n+1}}
$$

is a complex, 2D multipole expansion coefficient. Here, $|\underline{z}| \equiv \sqrt{z z^{*}}$ and $\operatorname{sgn}(n)$ denotes the sign of the integer $n$. Note that interior multipole expansion terms are labeled by positive integer $n$, whereas exterior terms are labeled by negative integer $n$. Terms labeled by $n$ correspond to $2|n|$-pole multipole field harmonics in $2 \mathrm{D}$. For example, $\underline{b}_{1}$ corresponds to the interior dipole moments, $\underline{b}_{2}$ the interior quadrupole moments, $\underline{b}_{3}$ the interior sextupole moments, etc.

Consistent with the constraints of commercially available material, we analyze the case of systems constructed from uniformly magnetized PM blocks. For a single such block with remanent field magnitude $B_{r}$ and an easy-axis that makes an angle $\beta$ with the $x$-axis, $\underline{B}_{r} \equiv B_{r} e^{i \beta}$ is constant within the block and can be taken outside the integral over the block area in Eq. (6). Then integrating once over $x$, and then once over $y$ and combining with the previous expression, one can obtain the multipole coefficients in several equivalent forms as $[2,4]$

$$
\underline{b}_{n}=\operatorname{sgn}(n) \frac{B_{r} e^{i \beta}}{2 \pi i} \oint \frac{d x}{\underline{z}^{n}}=\operatorname{sgn}(n) \frac{B_{r} e^{i \beta}}{4 \pi i} \oint \frac{d \underline{z}^{*}}{\underline{z}^{n}} .
$$

Here, $\oint d x \cdots$ and $\oint d \underline{z}^{*} \ldots$ are positive sense line integrals around the perimeter of the block.

For a system of uniformly magnetized PM blocks, linear superposition of the fields due to individual blocks as given by Eqs. (5) and (7) obtains the field of the assembly. It is often the case that the geometric location of a magnet block is given as a physical rotation 
of a reference block by an angle $\alpha$ about the origin. For such a block with an easy-axis that makes an angle $\beta+\gamma$ with the $x$-axis (after rotation), if follows from Eq. (6) that the multipole coefficient of the block is related to the reference block as [4]

$$
\underline{b}_{n}(\alpha, \gamma)=\underline{b}_{n}(0,0) e^{i[\gamma-\alpha(n+1)]}
$$

where $\underline{b}_{n}(0,0)$ denotes the multipole coefficient of the reference block.

In practical PM systems, it can be necessary to compensate for construction and material errors to achieve required field tolerances. Typically, tolerances on machining and mechanically positioning PM blocks ( \pm 1 mil possible) are such that the dominant source of field errors are associated with material errors in the PM medium. The most significant material errors of a uniformly magnetized PM block are errors in the angular orientation of the easy-axis $\left( \pm 2^{\circ}\right.$ typical) and in the remanent field strength ( $\pm 2 \%$ typical) $[6,7]$. Such errors typically dominate errors due to PM nonlinearities, etc. There are many possible methods to compensate for these material errors. Included among them are positioning small shim magnets, grinding sections of magnet blocks, sorting the magnet blocks into groups with compensating errors, and the introduction of small, movable thin strips of soft steel. But often the most attractive method is to tune the errors by making small changes in the positions of individual magnet blocks. For this purpose, and for estimating expected field errors, we summarize first-order perturbation effects for a uniformly magnetized magnet block. Let $\delta B_{r}$ and $\delta \beta$ be perturbations in the remanent field magnitude and easy-axis angle of the block, $\delta \underline{z}=\delta x+i \delta y$ be a perturbed linear displacement of the block by $\delta x$ and $\delta y$ along the $x$ - and $y$-directions with $\underline{B}_{r}$ fixed in the block, and $\delta \alpha$ be a perturbed angular rotation of the block about the origin with $\underline{B}_{r}$ fixed in the block. Then the corresponding first-order perturbations $\delta \underline{b}_{n}$ on the unperturbed block multipole coefficient $\underline{b}_{n}$ are easily obtained from Eq. (7) as [4]

$$
\delta \underline{b}_{n}=\left[\frac{\delta B_{r}}{B_{r}}+i(\delta \beta-n \delta \alpha)\right] \underline{b}_{n}-n \delta \underline{z} \underline{b}_{n+1}
$$

Note that the perturbed block displacement $\delta \underline{z}=\delta x+i \delta y$ causes a cascade of higher 
order multipoles into lower order multipoles as when the coordinate center of the multipole expansion is changed.

\section{MULTIPOLE MAGNETS WITH CONTINUOUS EASY-AXIS ORIENTATION}

When designing PM multipole magnets, we consider configurations of magnets that maximize one (or several) fundamental interior multipole coefficients and eliminate or hold all other interior multipole terms (error fields) and contaminating external fields to acceptable magnitudes. Continuous models where the PM material fills a region and has a continuously varying easy-axis angle and fixed remanent field magnitude allow one to determine geometries that ideally meet these requirements [2]. Such continuous magnets cannot be constructed due to material fabrication limits, but nevertheless, continuous models provide a guide to constructing practical segmented magnets to be discussed. Furthermore, they provide simple scaling relations approximately valid for practical segmented magnets, and thereby provide a convenient tool to determine regimes of applicability, etc. Here we present a continuous model based on an elliptically shaped distribution of PM material [8].

Consider the geometry illustrated in Fig. 2, where the PM material is contained within a region bounded by an inner ellipse with major and minor radii $a$ and $b$ aligned along the $x$ - and $y$-axes (i.e., $x^{2} / a^{2}+y^{2} / b^{2}=1$ with $a \geq b$ ) and a nested outer ellipse with major and minor radii scaled by a factor $s>1$. We choose the remanent field specified by

$$
\underline{B}_{r}[\underline{z}]=B_{r x}+i B_{r y}=B_{r} e^{i[\beta+(N+1) \psi]}
$$

in the PM material. Here, $B_{r}$ is the constant (real) magnitude of the remanent field, $N$

a positive integer, $\beta=$ const is a real phase-factor that sets the reference angle of the remanent field, and the angular variable $\psi$ is related to the azimuthal angle $\theta$ by $\tan \theta=$ $(b / a) \tan \psi$. This corresponds to a magnetization constant in magnitude and varying in angular orientation along each radial vector emanating from the origin $\underline{z}=0$. Throughout 
the entire elliptical regions interior to the aperture $\left(x^{2} / a^{2}+y^{2} / b^{2}<1\right)$ and exterior to the magnet $\left(x^{2} / a^{2}+y^{2} / b^{2}>s^{2}\right)$, the field resulting from this configuration can be expressed as [8]

$$
\begin{aligned}
\underline{B}^{*} & =B_{r} e^{i \beta} \sum_{n}^{\prime} \frac{\operatorname{sgn}(n)}{n-1}\left[1-\frac{1}{s^{n-1}}\right] F_{n}[a / b] \\
& \times\left(\begin{array}{c}
-(n+1) \\
(N-n) / 2
\end{array}\right)\left(\frac{a-b}{a+b}\right)^{(N-n) / 2}\left(\frac{z}{a}\right)^{n-1}
\end{aligned}
$$

where $\sum_{n}^{\prime}$ denotes a restricted sum carried out over a cascade of nonvanishing terms labeled by positive $n$ for the interior solution and negative $n$ for the exterior solution,

$$
F_{n}[a / b] \equiv 2^{n+1} n \frac{(a / b)^{n}}{[1+a / b]^{n+1}}
$$

is a dimensionless amplitude, and $\left(\begin{array}{l}m \\ p\end{array}\right)$ is a binomial coefficient defined for integer $m$ (positive, negative, or zero) and positive integer $p$ as

$$
\left(\begin{array}{c}
m \\
p
\end{array}\right) \equiv \begin{cases}\frac{m(m-1)(m-2) \cdots(m-p+1)}{p(p-1)(p-2) \cdots 1}, & m \geq p \text { or } m<0 \\
0, & 0 \leq m<p\end{cases}
$$

For the interior solution, the restricted sum is over the finite even cascade $n=2,4,6, \ldots$, $N$ for $N$ even, and the finite odd cascade $n=1,3,5, \ldots, N$ for $N$ odd. For the exterior solution, the sum is over the infinite cascade $n=-(N+2),-(N+4),-(N+6), \ldots, \infty$ for both even and odd $N$. For the special case of $n=1$ the limit $n \rightarrow 1$ should be taken, effectively replacing $1 /\left.(n-1)\left(1-1 / s^{n-1}\right)\right|_{n=1} \rightarrow \ln s$ in Eq. (11). This and other similar replacements are implicit in all formulae that follow.

For the special cases of $N=1$ and $N=2, \mathrm{Eq}$. (11) leads to single term field solutions interior to the aperture corresponding to pure dipole and quadrupole fields, respectively. For $N=1$ the interior dipole field is uniform within the aperture and given by

$$
\underline{B}^{*}=B_{r} e^{i \beta} F_{1}[a / b] \ln s
$$

while the field outside the magnet has external sextupole $(n=-3), 10$-pole $(n=-5), \ldots$ terms. Similarly, for $N=2$ the interior quadrupole field is given by 


$$
\underline{B}^{*}=B_{r} e^{i \beta} F_{2}[a / b]\left(1-\frac{1}{s}\right) \stackrel{\underline{z}}{a}
$$

while the field outside the magnet has external octupole $(n=-4), 12$-pole $(n=-6), \ldots$ terms. Note that $\left|\underline{B}^{*}\right|$ of the quadrupole field increases linearly within the aperture with distance from $\underline{z}=0$, and the maximum field magnitude is at the farthest aperture extent at $\underline{z}= \pm a$. Higher order $N \geq 3$ with $a \neq b$ will necessarily yield cascades of lower-order field components within the aperture and higher-order field components outside the aperture. For example, $N=3$ will yield internal sextupole and dipole field components and external 10-pole, 12-pole, $\ldots$ field components. For the special case of a circular magnet with $a=b$, all interior and exterior field components in Eq. (11) vanish except the fundamental $n=N$ term, yielding a pure $2 N$-pole field interior to the aperture with

$$
\left.\underline{B}^{*}\right|_{a=b}=B_{r} e^{i \beta} \frac{N}{N-1}\left[1-\frac{1}{s^{N-1}}\right]\left(\frac{z}{a}\right)^{N-1},
$$

and exactly zero field exterior to the màgnet. This is the familiar result for a perfect circular aperture multipole magnet obtained in ref. [2].

Note from Eq. (11) that the aperture field as measured by $\left|\underline{B}^{*}\right|$ at the maximum aperture extent at $\underline{z}= \pm a$ is independent of the absolute size of the system and the same $s$-dependent factor $\left(1-1 / s^{N-1}\right) /(N-1)$ appears in both circular $(a=b)$ and elliptical $(a>b)$ dipole $(N \rightarrow 1)$ and quadrupole $(N=2)$ magnets. It follows that an "equivalent" elliptical dipole or quadrupole magnet with the same remanent field magnitude $B_{r}$ and magnet surface scale factor $s$ as a circular $(a=b)$ dipole or quadrupole magnet has a larger aperture field for ellipticities $a / b$ where the amplitude satisfies $F_{N}[a / b]>F_{N}[a=b]=N$ and vice-versa when $F_{N}[a / b]<N$.

The dipole amplitude $F_{1}[a / b]=4(a / b) /[1+(a / b)]^{2}$ illustrated in Fig. 3(a) has a maximum value of unity at $a / b=1$ and decreases monotonically with increasing $a / b$. Since $F_{1}[a / b]<$ $F_{1}[1]=1$ for all ellipticities $a / b>1$, magnitude of the constant dipole field within the elliptical aperture magnet $(a>b)$ will be smaller than an "equivalent" circular dipole magnet $(a=b)$ with the same $B_{r}$ and $s$ by the factor $F_{1}[a / b]<1$. On the other hand, the quadrupole 
amplitude $F_{2}[a / b]=16(a / b)^{2} /[1+(a / b)]^{3}$ illustrated in Fig. $3(\mathrm{~b})$ is greater than the circular limit $(a=b)$ value of $F_{2}[1]=2$ over a range of ellipticities $1<a / b<2+\sqrt{5} \simeq 4.236$. Thus over this wide range of ellipticities, we find the surprising fact that the aperture field amplitude $\left|\underline{B}^{*}\right|_{\underline{z}= \pm a}$ of an elliptical aperture quadrupole magnet $(a>b)$ will be larger than an "equivalent" circular quadrupole magnet $(a=b)$ by the factor $F_{2}[a / b] / 2>1$. Conversely, for $a / b>2+\sqrt{5}, F_{2}[a / b]<2$ and $F_{2}$ is a monotonic decreasing function of $a / b$. Thus over this highly elliptical range, elliptical quadrupole magnets will have a weaker maximum field strength than equivalent circular magnets. Note that $F_{2}[a / b]$ has a maximum value of $F_{2}=64 / 27 \simeq 2.37$ at $a / b=2$, and therefore the maximum quadrupole field of the elliptical magnet is $\left|\underline{B}^{*}\right|_{\underline{z}= \pm a} \simeq 2.37 B_{r}(1-1 / s)$ rather than the familiar value of $\left|\underline{B}^{*}\right|_{r= \pm a}=2 B_{r}(1-1 / s)$ for the circular magnet.

\section{SEGMENTED MULTIPOLE MAGNETS}

So-called segmented multipole magnets are constructed from regular arrays of uniformly magnetized blocks of PM material and can therefore be built with commercially available materials. A variety of practical segmented configurations can be obtained by discretizing a continuous model $[2,3]$. For fine discretizations these generally result in magnets with field strength and quality less than, but approaching the continuous model. Here we summarize results obtained from segmenting the continuous elliptical magnet model in the circular limit $a=b=r_{i}$. Several practical multipole configurations obtained are presented. More complicated results with qualitatively similar features follow from..the segmentation of the continuous elliptical magnet with $a \neq b[8]$. Highly elliptical segmented dipole and quadrupole magnets appear attainable with good field quality and aperture field strengths on the same order as, or even stronger than segmented circular aperture magnets. These magnets promise to increase the range of applicability of iron-free PM systems in situations where an extended field region is only needed along one transverse dimension of the magnet aperture. In such situations, elliptical magnets can result in significant savings in magnet 
volume, possibly offsetting the increased cost associated with their greater complexity. Elliptical magnets will also have a decreased axial fringe field in comparison to circular magnets and may prove advantageous in applications with severe space limitations.

Simple segmented magnets with a fundamental field harmonic $N$ and circular clearbore apertures can be formed from $M \geq N$ magnet blocks of identical geometric shape. Individual magnet blocks are labeled with the index $j=1,2, \cdots, M$. The $j$ th block is located by rotating a reference block by an angle

$$
\alpha_{j}=\frac{2 \pi}{M}(j-1)+\Delta \alpha
$$

about the origin $\underline{z}=0$. Here, $\Delta \alpha=$ const is an arbitrary segmentation phase. The magnet blocks are uniformly magnetized, all with a common remanent field strength $B_{r}=$ const and the easy-axis of the $j$ th block makes an angle $\beta+\gamma_{j}$ with the $x$-axis, where $\beta$ is the easy-axis angle of the reference block and

$$
\gamma_{j}=(N+1) \alpha_{j}
$$

This $j$ th block easy-axis angle corresponds to the angular orientation of the continuous model at the azimuthal angle $\theta=\alpha_{j}$ ffor $a=b$ Eq. (10) becomes $\left.\underline{B}_{r}[\underline{z}]=B_{r} e^{i[\beta+(N+1) \theta]}\right\}$. Note that from one block to the next, the easy-axis advances by an angle of $(N+1) 2 \pi / M$ in the $x-y$ coordinate system, and by an angle of $N 2 \pi / M$ in a coordinate system of fixed orientation in each magnet block. Superimposing the fields due to individual blocks as given by Eqs. (5) and (8) and summing the resulting geometric series, one can express the unperturbed multipole field of the segmented magnet as $[2,4]$

$$
\underline{B}^{*}=M \sum_{\nu=0}^{\infty} \underline{b}_{n}(0,0) e^{-i \Delta \alpha(n-N)} \underline{\tilde{z}}^{n-1},
$$

where $\underline{b}_{n}(0,0)$ is the multipole coefficient of the reference block, and $n$ satisfies the selection rules

$$
n=N \pm \nu M
$$


where $\nu=0,1,2,3, \cdots$, and the + sign applies to the interior $\left(r<r_{i}\right)$ solution and the sign applies to the exterior $\left(r>s r_{i}\right)$ solution. The interior field described by Eqs. (18) and (19) has a fundamental term $M \underline{b}_{N}(0,0) \underline{z}^{N-1}$, and the first interior error field component is the term $M \underline{b}_{N+M}(0,0) e^{-i M \delta \alpha} \underline{z}^{N-1+M}$. For reasonably large $M$ and appropriately shaped blocks this interior expansion will be dominated by the fundamental term, and the interior error field terms with $n=N+\nu M$ and $\nu \geq 1$ have high-order radial powers and will decrease rapidly in magnitude with decreasing $r$ within the aperture. Also, for most usual parameters the exterior field is high-order and shielding should not be necessary for most applications. The intrinsic high- $n$ interior error terms allowed by the selection rule (19) can be controlled by using a sufficiently large aperture, and as a practical matter, lower-harmonic interior error fields resulting from construction and materials errors will typically be more troublesome. Analogously to Eq. (18), the multipole expansion of the first-order error field can be expressed as [4]

$\delta \underline{B}^{*}=\sum_{n} e^{-i \Delta \alpha(n-N)} \sum_{j=1}^{M}\left\{\left[\frac{\delta B_{r j}}{B_{r}}+i\left(\delta \beta_{j}-n \delta \alpha_{j}\right)\right] \underline{b}_{n}(0,0)-n \delta \underline{z}_{j} \underline{b}_{n+1}(0,0)\right\} e^{-i 2 \pi(n-N)(j-1) / M} \underline{z}^{n-1}$

where the sum is over positive/negative integer $n$ for the interior/exterior expansions, and $\delta B_{r j}, \delta \beta_{j}, \delta \alpha_{j}$, and $\delta \underline{z}_{j}$ are perturbations of the $j$ th block defined with respect to the reference block. This equation can be used both to evaluate expected field errors and to determine changes in block positions for their correction.

Explicit formulae for the reference block multipole coefficients $\underline{b}_{n}(0,0)$ are readily calculated for a variety of geometries using Eq. (7). The simple geometries illustrated in Fig. 4 with circular, rectangular, and trapezoidal block shapes are practically useful and lead to the following expressions $[2,4]$

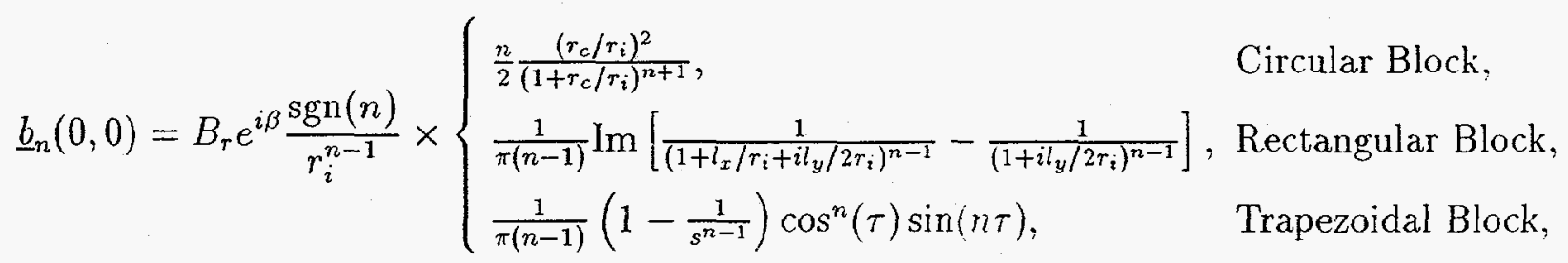

where $\operatorname{Im}[\cdots]$ denotes the imaginary part of $\cdots$, i.e., $\operatorname{Im}[x+i y]=y$. All blocks are centered as indicated on the $x$-axis, are inscribed within the annular region $r_{i} \leq r \leq s r_{i}$ with $s>1$, and 
subtend a angular half-width of $\tau$ about the origin. Note that $\tau$ and $s$ are not independent for the circular block, i.e., the rod radius $r_{c}$ satisfies $r_{c} / r_{i}=\sin \tau /(1-\sin \tau)=(s-1) / 2$. Also, the $x$ - and $y$-widths of the rectangular block are $\ell_{x}=r_{i}(s-1)$ and $\ell_{y}=2 r_{i} \tan \tau$. For the special case of $n=1$, the limit $n \rightarrow 1$ is taken in the rectangular and trapezoidal block expressions.

Cost effective segmented multipole magnets achieve good field quality and high aperture fields with a minimal volume of PM material (except for ferrites, the medium is expensive) and have a small number of distinct magnet pieces of simple shape to control costs associated with making and assembling a large number of parts. As a general rule, the field strength given by the continuous easy-axis model [see Eq. (15) with $a=r_{i}$ ] provides an upper bound to the achievable field strength of a segmented magnet with PM material confined within the annular region $r_{i} \leq r \leq s r_{i}$. The better the segmented magnet approximates the symmetry of the continuous model, and the more the PM material fills the annular region, the better the field quality and the larger the aperture field strength of the magnet will be.

The reference block multipole expressions (21) together with Eqs. (16)-(20) are straightforward to apply to develop and analyze the fields from a variety of practical multipole magnets [2]. Several representative quadrupole magnets $(N=2)$ are presented in Fig. 5 . Arrows indicate the direction of the magnetization in each magnet piece and a remanent field phase of $\beta=3 \pi / 2$ is chosen so the principal axes of the quadrupole field align with the $x$ - and $y$-coordinate axes and $d B_{y} / d x>0$. In Fig. $5(\mathrm{a})$ an $M=8$ segment square-block quadrupole needing only one distinct magnet piece is shown for a block half angular width of $\tau=0.8 \times \pi / 8$ and a segmentation phase of $\Delta \alpha=0$. As drawn, this magnet will have a fundamental aperture field strength at $r=r_{i}$ of $B_{\tau} \times 0.456$. In Fig. $5(\mathrm{~b})$ an $M=16$ segment trapezoidal-block magnet is shown with $\tau=\pi / M$ for maximum magnet packing and a segmentation phase of $\Delta \alpha=\pi / M$, so only 4 distinct magnet pieces are needed (rather than 5 with $\Delta \alpha=0$ ). As drawn, this magnet will have a higher aperture field strength of $B_{r} \times 0.937$.

Properties of the magnets in Fig. 5 along with numerous other practical multipole mag- 
nets are summarized in Tables I-III. Magnets obtained from square $\left(\ell_{x}=\ell_{y}\right)$, rectangular $\left(\ell_{x} \neq \ell_{y}\right)$, and trapezoidal blocks are each tabulated for dipole $(N=1)$, quadrupole $(N=2)$, and sextupole $(N=3)$ configurations. Included for each magnet type is a scaled expression for the interior $\left(r<r_{i}\right)$ multipole field expansion (18) with magnet block parameters given in terms of the half-angular width $\tau$ of each block and (for rectangular and trapezoidal blocks) the outer surface scaling factor $s$. For each specific configuration with fundamental harmonic $N$ and $M$ magnet blocks, the following are tabulated: the number of distinct magnet types needed for the indicated remanent field and segmentation phases $\beta$ and $\Delta \alpha$; an expression for the field amplitude of the fundamental harmonic at the aperture radius $r=r_{i}$; and an upper bound for the amplitude. Only economical configurations leading to a minimal number of distinct magnet blocks are tabulated. The fundamental field amplitude is given in terms of the remanent field magnitude $B_{r}$, the block half-angular width $\tau(0 \leq \tau \leq \pi / M)$, and (for rectangular and trapezoidal blocks) the outer surface scaling factor $s(s \geq 1)$. The maximum field amplitudes correspond to block half-angular widths of $\tau=\pi / M$ (i.e., adjacent blocks just touch), except for several small $M$ trapezoidal block configurations marked with $\diamond$, where the indicated maxima occur $\tau$ a bit less then the geometric limit $\tau=\pi / M$. For simplicity, the maximum amplitudes of the rectangular block configurations in Table II are given in the infinite block limit with $s \rightarrow \infty$. Achievable field magnitudes with finite $s$ will be somewhat less than these limiting values.

Tables I-III summarize a large number of configurations leading to practical multipole magnets. Various design possibilities can be rapidly evaluated with the information provided. Limiting field strengths, components needed, and intrinsic field errors are all easily determined. Due to the independence of. the aperture $\left(r=r_{i}\right)$ field harmonics on the physical size of the magnets, note that numerous compact, high gradient structures are possible. Also, the amplitude of intrinsic field errors due to the harmonics $N+\nu M$ with $\nu \geq 1$ decrease rapidly within the aperture due to the $\left(r / r_{i}\right)^{\nu M}$ factors, indicating good intrinsic field quality within a so-called "good-field" radius that will be close to the aperture radius.

Several useful observations pertaining to Tables I-III can be made. For given values of 
$s, M$, and $B_{r}$, the maximum field strength of the trapezoidal configurations, which better approximate circular symmetry, are larger than for the square or rectangular block configurations. Thus, for a given aperture field strength, a trapezoidal configuration will always be more compact than square or rectangular configurations. Also, trapezoidal configurations are capable of achieving higher aperture fields, and for large $M$ approach the continuous limit field strength given by Eq. (15) with $a=r_{i}$ [2]. However, since PM material is generally produced in rectangular blocks which can be cut into specific shapes, square and rectangular magnet blocks can result in little material waste in comparison to trapezoidal blocks, and therefore will frequently prove to be more economical. Thus, square or rectangular block configurations are more attractive for low aperture fields, whereas for high fields or in situations with severe space limitations, trapezoidal block configurations are generally appropriate. For a given block type and fundamental harmonic $N$, more magnet blocks $M$ will yield better intrinsic field quality. For rectangular and trapezoidal blocks, higher $M$ will also result in larger aperture fields, whereas for square blocks larger field strengths will be obtained for small or intermediate $M$ due to decreasing square magnet size with increasing $M$. Finally, for rectangular and trapezoidal configurations, one finds that for any value of the magnet surface scaling factor $s$ that the first error field harmonic $N+M$ can be made to vanish through an appropriate choice of block half-angular width $\tau$, thereby increasing the intrinsic field quality [2]. This value of $\tau$ will be slightly less than $\tau=\pi / M$, corresponding to small gaps between neighboring blocks.

Multipole magnets can also be produced from arrays of circular magnet blocks with a common radius and remanent field strength. The advantage of such structures are that only a single magnet type is necessary for any fundamental harmonic, since any magnetization angle can be obtained by rotating a block about its axis. However, significant amounts of PM material will be lost when fabricating circular rods and only modest aperture field strengths will be achievable, rendering such configurations attractive only for low field strengths or perhaps for purposes of error field tuning of other magnet arrays. Employing the same procedures and notation as above, the multipole expansion of a rod magnet array is given 
by [4]

$$
\begin{aligned}
\underline{B}^{*}=B_{\tau} e^{i \beta} \frac{M}{2} \sum_{\nu=1}^{\infty} & e^{i \Delta \alpha(n-N)} n \sin ^{2}(\tau) \\
& \times(1-\sin \tau)^{n-1}\left(\frac{z}{r_{i}}\right)^{n-1},
\end{aligned}
$$

where $n=N+\nu M$. Excepting tuning applications, it will be advantageous to employ configurations with as many rods $M$ as possible to achieve the required aperture field strength $B_{r}(M N / 2) \sin ^{2}(\tau)(1-\sin \tau)^{N-1}$ with $\tau$ approaching the limiting value $\tau=\pi / M$, and thereby minimize the waste of PM material and provide higher field quality.

As a practical matter, nonlinear self-demagnetization effects should be evaluated before a particular configuration is deemed practical. The threshold for such effects depends on the particular PM material employed. Generally, demagnetization effects will be an issue in magnet blocks where the applied field $\mu_{0} \mathrm{H}$ is driven significantly antiparallel to the remanent field $\mathbf{B}_{r}$. Avoiding such material thresholds can limit the achievable magnet field strength. For example, due to the $\ln s$ factors in the dipole magnet strengths in Table III, one might naively conclude that arbitrarily large dipole fields are possible by letting magnet surface scaling factor $s$ become large. However, the constant dipole field within the aperture will be antiparallel (or nearly so) to $\mathbf{B}_{r}$ near the aperture surface of at least one magnet block. Thus if a PM material is used where the onset of demagnetizing effects occurs on entry to the "third quadrant" of the $B_{\|}\left(H_{\|}\right)$material characteristics curve (i.e., at $\mu_{0} H_{\|}=-B_{r}$ ), then magnet sizes must be chosen such that the dipole field strength does not exceed $B_{\tau}$. Detailed evaluation of demagnetization effects requires calculation of the fields within individual magnet blocks and is beyond the scope of this paper. Calculations of such interior fields must be done with great care if discrete numerical methods are employed, but fortunately, analytical methods can be employed to exactly perform the needed evaluations.

Finally, in situations where high field quality is desired, it will often be necessary to compensate for material errors. As an illustrative example, we consider an $M=8$ segment square block quadrupole $(N=2)$ magnet [see Fig. 5(a)]. The block half-angular width is taken to be $\tau=0.86 \times \pi / 8$ radians, corresponding to a 0.500 Tesla field strength at 
the aperture radius $r=r_{i}$, and the first intrinsic error field harmonic is $n=10$ with an amplitude of $3.05 \%$ of the fundamental at the aperture radius. This leading order intrinsic error harmonic will have an amplitude of less than $0.2 \%$ of the fundamental within a good field radius of $r=0.7 r_{i}$, indicating good intrinsic field quality. Magnet blocks are assumed to have uniformly distributed errors in easy-axis angle $\left( \pm 2^{\circ}\right)$ and remanent field strength $( \pm 2 \%)$. Mechanical errors are neglected and it is found from Eq. (20) that low-n error field harmonics result and the leading-order rms amplitudes of the first few harmonics are $0.56,0.82,0.89,0.84,0.72,0.58,0.42$, and 0.27 percent of the unperturbed fundamental at the aperture radius for $n=1,2,3, \cdots 8$, respectively. For this $M=8$ square block quadrupole, these low-harmonic field errors can be tuned by shifting the radial positions of individual magnet blocks. One possible procedure is as follows: Since both the magnitude and phase errors in the fundamental cannot be simultaneously tuned by simple radial block movements [see Eq. (20)], all blocks are first moved radially in or out an equal distance until the fundamental has the correct magnitude in the presence of magnet errors. Then individual radial perturbations are found for each block about this tuned aperture radius [i.e., $\delta \underline{z}_{j}=\delta x_{j} \equiv \delta r_{j}$ in the reference block position indicated in Fig. 4(b)] to zero the $n=3$ and $n=4$ error field harmonics to first-order, subject to the constraint that $\sum_{j=1}^{M} \delta r_{j}=0$, so the fundamental harmonic remains unchanged by the radial perturbations. It is found that these eight perturbations in radial block location are sufficient to eliminate the $n=3$, 4 and 5 harmonics to first-order for all possible errors. Appropriate perturbations $\left\{\delta r_{j}\right\}$ are straightforward to calculate from Eq. (20) for any particular set of magnet block errors using a singular value decomposition. One finds that the leading-order rms amplitudes of the field errors at the untuned aperture radius $r=r_{i}$ are 0.38 percent of the fundamenta! for $n=1,0$ for $n=3,4$, and 5 , and $0.29,0.39$, and 0.25 percent of the fundamental for $n=6,7$, and 8 . Furthermore, note that the magnitude of the total $n=2$ quadrupole harmonic is correct by construction, but will in general have a phase error. After this tuning of individual radial block locations, the coordinate center can be translated a small distance to eliminate any dipole field component, and then these translated coordinate axes 
can be rotated about their center to correct any phase error in the fundamental quadrupole harmonic as measured within the new coordinate system. This procedure will leave the magnitude of the quadrupole field component unchanged to second-order in the translation. As measured in this new coordinate system, the leading-order field errors are zero for $n=1$, 2,3 , and 4 , and the rms aperture amplitudes are $0.01,0.28,0.39$ and 0.25 percent of the fundamental for $n=5,6,7$, and 8. These error harmonics correspond to field errors that are below $0.05 \%$ of the fundamental within a good field radius of $0.7 r_{i}$, thereby yielding a high field quality magnet. In practice, achievable error tuning will depend on many factors, including how accurately the radial positions of the blocks can be adjusted relative to the size of the aperture and on how accurately the field errors can be measured. Generally speaking, many other error field tuning procedures are possible $[2,4,7]$. For any particular configuration, one must be careful to adopt a procedure that allows all possible errors to be tuned to the desired order. Determination of appropriate classes of block displacements and other perturbations to achieve this can be nontrivial.

\section{ACKNOWLEDGMENTS}

This research was performed under the auspices of the U.S. Department of Energy by Lawrence Livermore National Laboratory under contract W-7405-ENG-48. One of the authors (S.M. Lund) was supported in part by an appointment to the U.S. Department of Energy Postdoctoral Research Program administered by the Oak Ridge Institute for Science and Education. 


\section{REFERENCES}

[1] "The Art and Science of Magnet Design," and references therein, PUB-754, Lawrence Berkeley Laboratory, University of California, February 1995.

[2] K. Halbach, "Design of Permanent Multipole Magnets with Oriented Rare Earth Cobalt Material," Nucl. Instr. and Meth. 169, 1 (1980).

[3] K. Halbach, "Physical and Optical Properties of Rare Earth Cobalt Magnets," Nucl. Instr. and Meth. 187, 109 (1981).

[4] K. Halbach, "Perturbation Effects in Segmented Rare Earth Cobalt Multipole Magnets," Nucl. Instr. and Meth. 198, 213 (1981).

[5] UGIMAG, Inc., Pechiney Group, 405 Elm Street, Valparaiso, IN 46383, USA.

[6] S. Herb, "Field Quality and Stability of Permanent Magnet Quadrupoles," IEEE Trans. Nuc. Sci. bf 32,3578 (1985).

[7] G.B. Bowden, "Permanent Magnet Quadrupole Field Errors," ABC Technical Note, Stanford Linear Accelerator Center, May, 1993.

[8] S.M. Lund and K. Halbach, "Wide Aperture Multipole Magnets from Elliptical Arrays of Permanent Magnets," to appear. 


\section{TABLES}

TABLE I. Configurations for square block multipole magnets.

TABLE II. Configurations for rectangular block multipole magnets.

TABLE III. Configurations for trapezoidal block multipole magnets. 


\section{FIGURES}

FIG. 1. Geometry and field structures of axially extruded PM systems, for 2D and semi-infinite magnets (a), and for a magnet of finite axial length $\ell$ as a superposition of two semi-infinite magnets (b).

FIG. 2. 2D elliptical magnet geometry. The PM material is shaded, and rectangular $x-y$ and cylindrical $r-\theta$ coordinates are defined as indicated.

FIG. 3. Plots of dipole (a) and quadrupole (b) magnet amplitudes $F_{1}[a / b]$ and $F_{2}[a / b]$ verses magnet ellipticity $a / b$.

FIG. 4. Reference magnet blocks of circular (a), rectangular (b), and trapazoidal (c) shape.

FIG. 5. Schematic cross-sections of segmented quadrupole magnets formed from $M=8$ square block magnets (a) and $M=16$ trapazoidal magnets. 
Square Block Multipoles

\begin{tabular}{|c|c|c|c|c|c|c|}
\hline & & $=B_{r} e^{i \beta} I$ & $M(M / \pi) \sum_{\nu=1}^{\infty} e^{-}$ & $-i \Delta \alpha(n-N$ & $\begin{array}{l}\operatorname{Im}\left[1 /(1+\{2+i\} \tan \tau)^{n-1}-1 /(1+i \tan \right. \\
\quad n=N+\nu M\end{array}$ & $\left.\tau)^{n-1}\right] /(n-1)$ \\
\hline & & & & Magnet & Aperture $\left(r=r_{i}\right)$ Value of Fundament & al Harmonic \\
\hline$N$ & $M$ & $\beta$ & $\Delta \alpha$ & Types & Formula & Max. $(\tau=\pi / M)$ \\
\hline & 4 & & & 1 & & $B_{r} \times 0.590$ \\
\hline & 6 & & & $\overline{2}$ & & $B_{r} \times 0.500$ \\
\hline 1 & 8 & arbitrary & $0, \pi / M$ & & $B_{r}(M / \pi)\{\tau-\arctan [\tan (\tau) /(1+2 \tan \tau)]\}$ & $B_{r} \times 0.433$ \\
\hline & \begin{tabular}{|l|}
10 \\
12 \\
\end{tabular} & & & 3 & & $\begin{array}{l}B_{r} \times 0.381 \\
B_{r} \times 0.340\end{array}$ \\
\hline & 4 & & $0 \leq \Delta \alpha \leq \pi / M$ & $\overline{1}$ & & $\overline{B_{\mathrm{r}} \times 0.509}$ \\
\hline 2 & $8:$ & arbitrary & & & $B_{r}(4 M / \pi)(1+\tan \tau) \sin ^{2}(\tau) /$ & $B_{r} \times 0.600$ \\
\hline & \begin{tabular}{|l|}
12 \\
16 \\
\end{tabular} & & $0, \pi / M$ & $\overline{2}$ & $\left(1+4 \tan \tau+5 \tan ^{2} \tau\right)$ & $\frac{B_{r} \times 0.534}{B_{r} \times 0.466}$ \\
\hline & 6 & & $0 \leq \Delta \alpha \leq \pi / M$ & $\overline{1}$ & & $\overline{B_{r} \times 0.524}$ \\
\hline & \begin{tabular}{|c|}
8 \\
\end{tabular} & & & $\overline{2}$ & & $B_{r} \times 0.612$ \\
\hline 3 & 9 & arbitrary & & & $B_{r}(M / \pi) \tan (\tau)\left[\cos ^{4} \tau\right.$ & $B_{r} \times 0.627$ \\
\hline & 12 & & $0, \pi / M$ & 1 & $\left.-(1+2 \tan \tau) /\left(1+4 \tan \tau+5 \tan ^{2} \tau\right)^{2}\right]$ & $B_{r} \times 0.625$ \\
\hline & \begin{tabular}{|l|}
18 \\
24 \\
\end{tabular} & & & 2 & & $\frac{B_{r} \times 0.556}{B_{r} \times 0.483}$ \\
\hline
\end{tabular}

$$
\text { Table I; Lund and Habach }
$$


Rectangular Block Multipoles

\begin{tabular}{|c|c|c|c|c|c|c|}
\hline \multicolumn{7}{|c|}{$\begin{array}{c}B^{*}=B_{r} e^{i \beta}(M / \pi) \sum_{\nu=1}^{\infty} e^{-i \Delta \alpha(n-N)} \operatorname{Im}\left[1 /(s+i \tan \tau)^{n-1}-1 /(1+i \tan \tau)^{n-1}\right] /(n-1) \\
n=N+\nu M\end{array}$} \\
\hline \multirow[b]{2}{*}{$\Lambda$} & & \multirow[b]{2}{*}{$\beta$} & \multirow[b]{2}{*}{$\Delta \alpha$} & \multirow[b]{2}{*}{$\begin{array}{l}\text { Magnet } \\
\text { Types }\end{array}$} & \multicolumn{2}{|c|}{ Aperture $\left(r=r_{i}\right)$ Value of Fundamental Harmonic } \\
\hline & $M$ & & & & Formula & $\begin{array}{c}\text { Maximum } \\
\tau=\pi / M, s \rightarrow \infty\end{array}$ \\
\hline & 4 & & $\pi / M$ & 1 & \multirow{6}{*}{$B_{r}(M / \pi)\{\tau-\arctan [\tan (\tau) / s]\}$} & \multirow{6}{*}{$0, \cdots, \ldots,-20$} \\
\hline & 6 & & $0, \pi / M$ & 2 & & \\
\hline & 8 & $0, \pi, \pm \pi / 2$ & $\pi / M$ & & & \\
\hline & 10 & & $0, \pi / M$ & $\overline{3}$ & & \\
\hline & 12 & & $\pi / M$ & & & \\
\hline & 16 & & & 4 & & \\
\hline \multirow{5}{*}{2} & 4 & \multirow{5}{*}{$0, \pi, \pm \pi / 2$} & \multirow{2}{*}{$0, \pi / M$} & \multirow[t]{2}{*}{1} & \multirow{5}{*}{$\begin{array}{c}B_{r}(M / 2 \pi) \sin (2 \tau)\left(s^{2}-1\right) / \\
\left(s^{2}+\tan ^{2} \tau\right)\end{array}$} & $B_{r} \times 0.637$ \\
\hline & \begin{tabular}{|l|}
6 \\
\end{tabular} & & & & & $B_{r} \times 0.827$ \\
\hline & 8 & & $\pi / M$ & 1 & & $B_{r} \times 0.900$ \\
\hline & 12 & & $\overline{0, \pi / M}$ & 2 & & $B_{r} \times 0.955$ \\
\hline & 16 & & $\pi / M$ & & & $B_{r} \times 0.974$ \\
\hline \multirow{6}{*}{3} & 6 & \multirow{6}{*}{$0, \pi, \pm \pi / 2$} & $\overline{0, \pi / \bar{M}}$ & $\overline{\overline{1}}$ & \multirow{6}{*}{$\begin{array}{c}B_{r}(M / \pi) \tan (\tau) \times \\
{\left[\cos ^{4} \tau-s /\left(s^{2}+\tan ^{2} \tau\right)^{2}\right]}\end{array}$} & $B_{r} \times 0.620$ \\
\hline & 8 & & $\pi / M$ & \multirow[t]{3}{*}{2} & & $B_{r} \times 0.768$ \\
\hline & 9 & & $\overline{0, \pi / M}$ & & & $B_{r} \times 0.813$ \\
\hline & 12 & & $\pi / M$ & & & $B_{r} \times 0.891$ \\
\hline & 18 & & $0, \pi / M$ & $\overline{2}$ & & $B_{r} \times 0.950$ \\
\hline & 24 & & $\pi / M$ & & & $B_{r} \times 0.972$ \\
\hline
\end{tabular}

\section{Table II, Lund and Haltach}


Trapezoidal Block Multipole

\begin{tabular}{|c|c|c|c|c|c|c|}
\hline & & $\underline{3}^{*}=B_{r} e^{i f}$ & $(M / \pi$ & $\sum_{\nu=1}^{\infty} e^{-}$ & $\begin{array}{c}-i \Delta \alpha(n-N)\left\{\left(1-1 / s^{n-1}\right) /(n-1)\right] \cos ^{n} \\
n=N+\nu M\end{array}$ & $\tau \sin (n \tau)\left(z / r_{i}\right)^{n-1}$ \\
\hline \multirow[b]{2}{*}{$N$} & \multirow[b]{2}{*}{$M$} & \multirow[b]{2}{*}{$\beta$} & \multirow[b]{2}{*}{$\Delta \alpha$} & \multirow{2}{*}{$\begin{array}{c}\text { Magnet } \\
\text { Types }\end{array}$} & \multicolumn{2}{|c|}{ Aperture $\left(r=r_{i}\right)$ Value of Fundamental Harmonic } \\
\hline & & & & & Formula & Maximum $(\tau=\pi / M)$ \\
\hline \multirow{5}{*}{1} & 4 & $\overline{0, \pi, \pm \pi / 2}$ & $\pi / M$ & 2 & \multirow{5}{*}{$B_{r}(M / 2 \pi) \sin (2 \tau) \ln s$} & \multirow{3}{*}{\begin{tabular}{|l}
$B_{r} \times 0.637 \ln s$ \\
$B_{r} \times 0.827 \ln s$
\end{tabular}} \\
\hline & \multirow{4}{*}{\begin{tabular}{|c|}
8 \\
12 \\
\end{tabular}} & $\pm \pi / 2$ & 0 & \multirow[t]{2}{*}{$\overline{3}$} & & \\
\hline & & $0, \pi$ & \multirow{3}{*}{$\pi / M$} & & & \\
\hline & & $0, \pi, \pm \pi / 2$ & & 4 & & \multirow{2}{*}{\begin{tabular}{|l|}
$B_{r} \times 0.900 \ln s$ \\
$B_{r} \times 0.955 \ln s$ \\
\end{tabular}} \\
\hline & & & & 6 & & \\
\hline & \multirow[t]{2}{*}{4} & $0, \pi$ & $\pi / \bar{M}$ & 1 & \multirow{10}{*}{$B_{\mathrm{r}}(M / \pi) \cos ^{2}(\tau) \sin (2 \tau)(1-1 / s)$} & \multirow[t]{2}{*}{$B_{r} \times 0.827(1-1 / s)^{\circ}$} \\
\hline & & $\pm \pi / 2$ & $\overline{0}$ & \multirow{3}{*}{2} & & \\
\hline & 6 & $0, \pi$ & $0, \pi / M$ & & & $B_{r} \times 1.240(1-1 / s)$ \\
\hline \multirow{7}{*}{2} & 8 & $0, \pi, \pm \pi / 2$ & $\pi / M$ & & & $\overline{B_{r} \times 1.537(1-1 / s)}$ \\
\hline & 10 & $0, \pi$ & $0, \pi / \bar{M}$ & \multirow{3}{*}{3} & & \multirow{3}{*}{\begin{tabular}{|l|}
$B_{r} \times 1.692(1-1 / s)$ \\
$B_{r} \times 1.782(1-1 / s)$
\end{tabular}} \\
\hline & 12 & & $\pi / M$ & & & \\
\hline & & $\pm \pi / 2$ & 0 & & & \\
\hline & \multirow{3}{*}{\begin{tabular}{|l|}
16 \\
20 \\
\end{tabular}} & $0, \pi, \pm \pi / 2$ & \multirow[t]{2}{*}{$\pi / \bar{M}$} & 4 & & \multirow{3}{*}{\begin{tabular}{|l}
$B_{r} \times 1.875(1-1 / s)$ \\
$B_{r} \times 1.919(1-1 / s)$
\end{tabular}} \\
\hline & & $0, \pi$ & & \multirow[t]{2}{*}{5} & & \\
\hline & & $\pm \pi / 2$ & 0 & & & \\
\hline \multirow{7}{*}{3} & \multirow[t]{2}{*}{6} & $\overline{0, \pi}$ & $\pi / \bar{M}$ & \multirow[t]{2}{*}{1} & \multirow{7}{*}{$B_{r}(M / 2 \pi) \cos ^{3}(\tau) \sin (3 \tau)\left(1-1 / s^{2}\right)$} & \multirow[t]{2}{*}{$B_{r} \times 0.696\left(1-1 / s^{2}\right)^{2}$} \\
\hline & & $\pm \pi / 2$ & 0 & & & \\
\hline & 9 & $0, \pi$ & $0, \pi / M$ & \multirow[t]{2}{*}{2} & & $\overline{B_{r} \times 1.029\left(1-1 / s^{2}\right)}$ \\
\hline & \multirow{3}{*}{$\frac{12}{18}$} & $\overline{0, \pi, \pm \pi / 2}$ & $\pi / M$ & & & $B_{r} \times 1.217\left(1-1 / s^{2}\right)$ \\
\hline & & $0, \pi$ & & $\overline{3}$ & & $B_{r} \times 1.368\left(1-1 / s^{2}\right)$ \\
\hline & & $\pm \pi / 2$ & $\overline{0}$ & & & \\
\hline & 24 & $0, \pi$ & $\pi / M$ & 4 & & $B_{r} \times 1.425\left(1-1 / s^{2}\right)$ \\
\hline
\end{tabular}

\section{Table III, Lund ane Halbach}




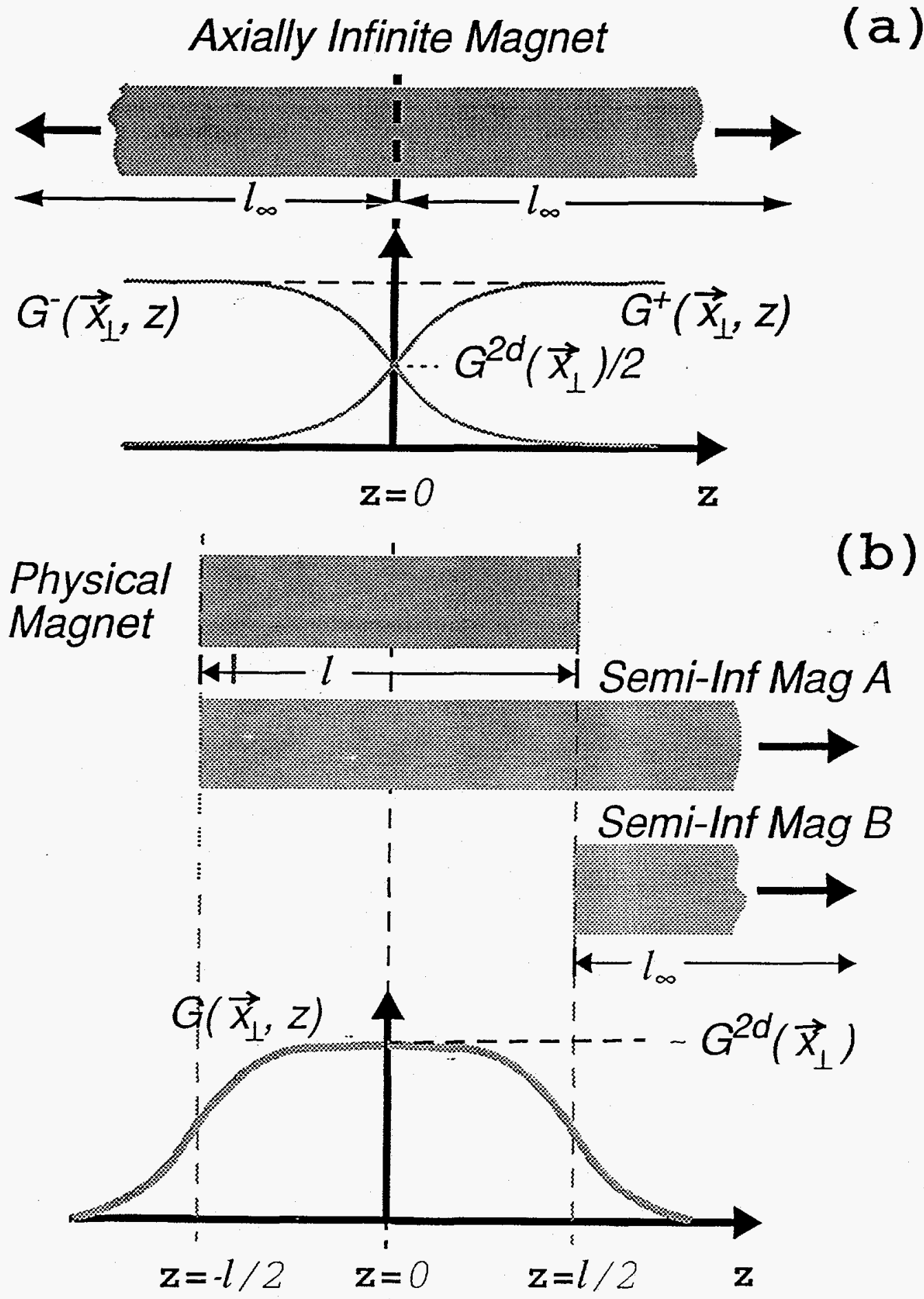




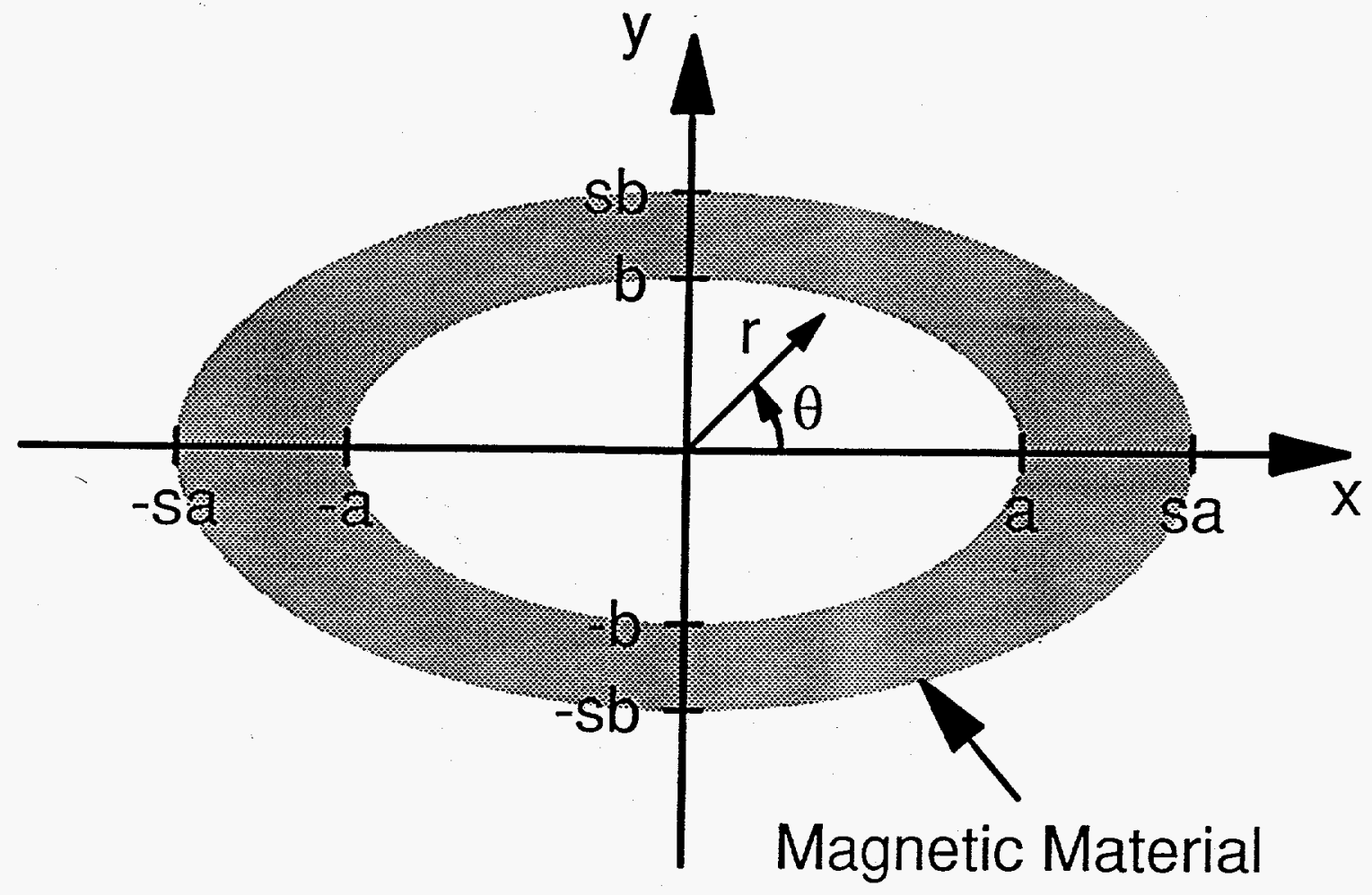



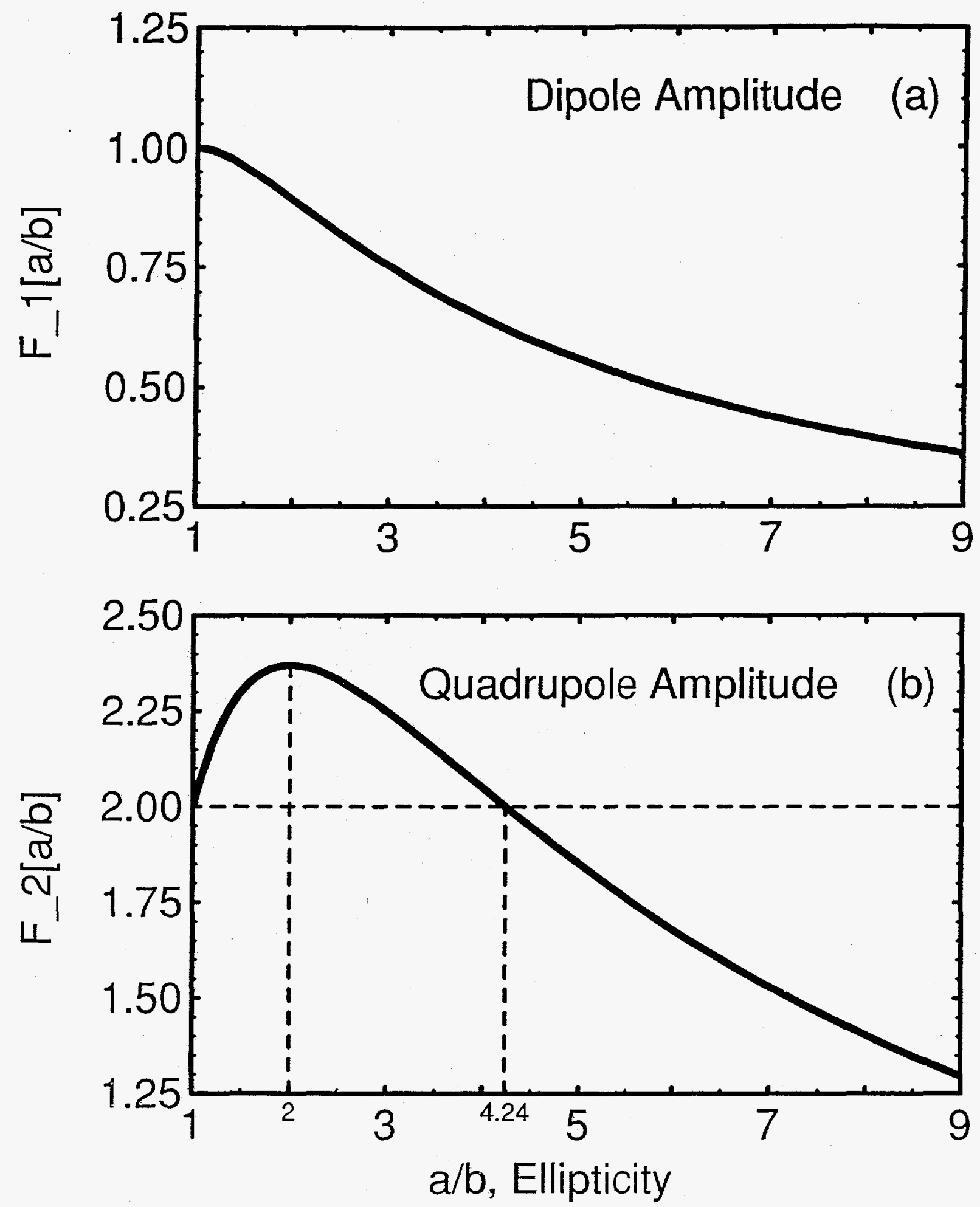


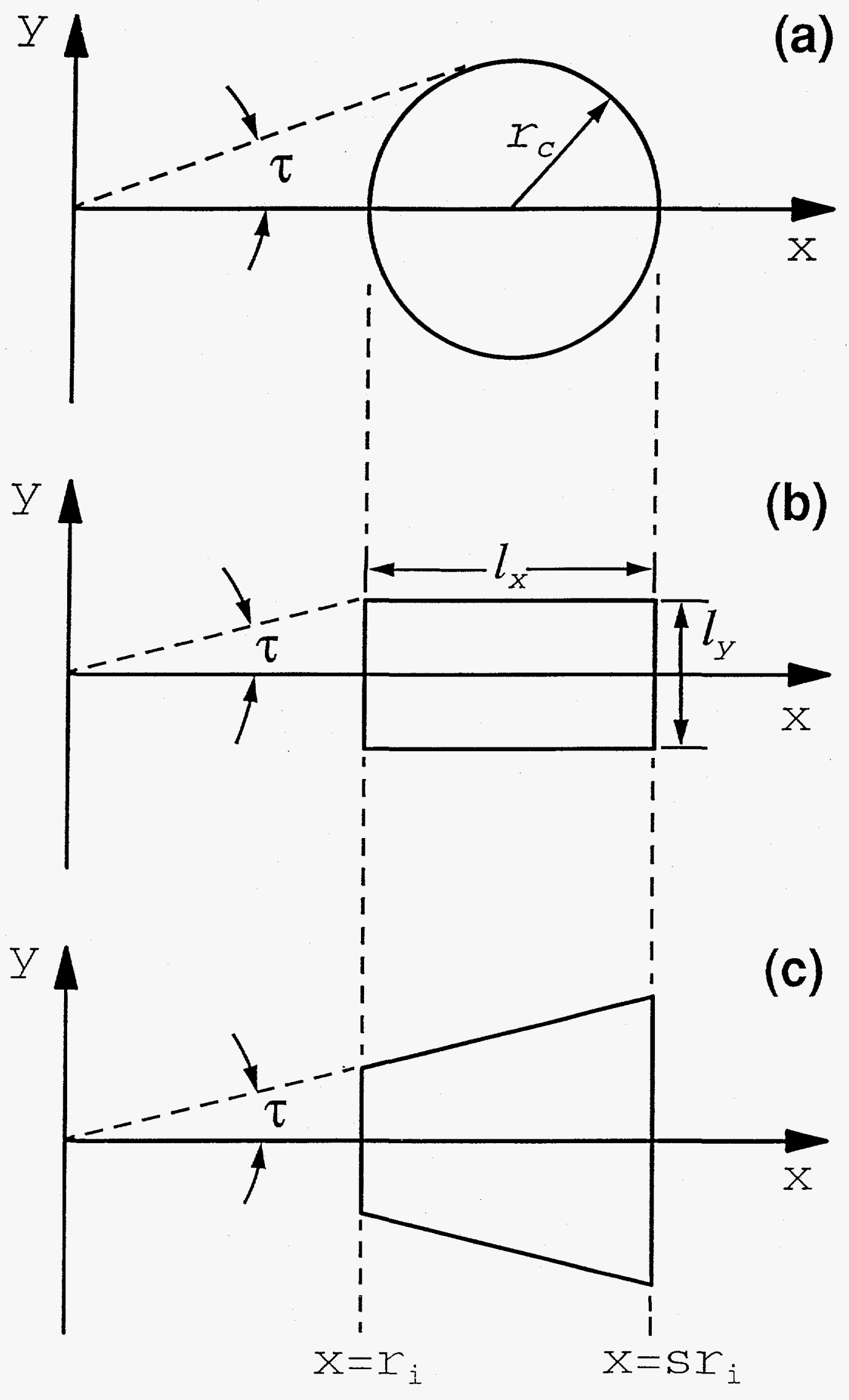



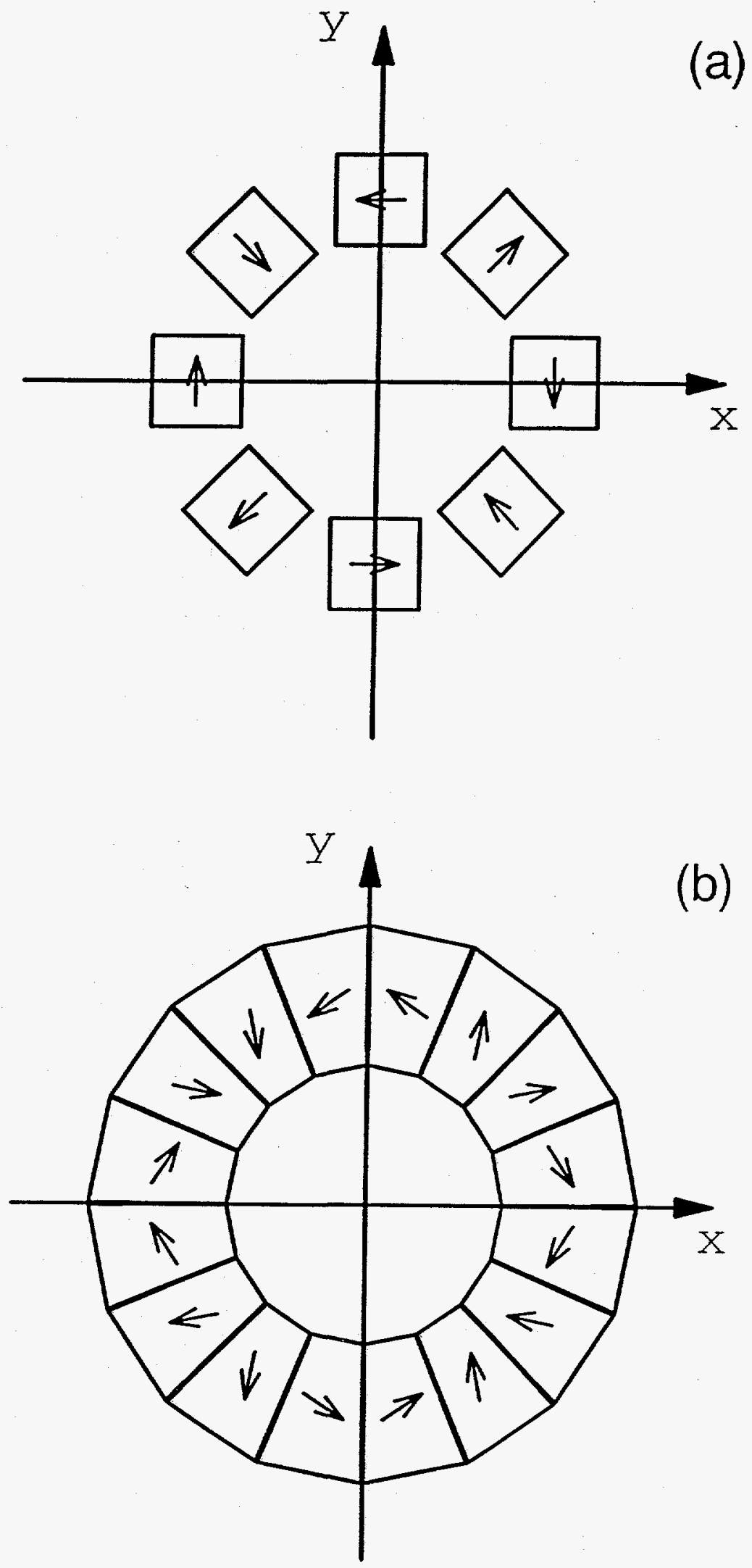